\section{Comments on the use of perceptually ambiguous figures as McCollough-effect stimuli}

\author{
JACK BROERSE \\ Mount Gravatt College of Advanced Education, \\ Mt. Gravatt, 4122, Australia \\ and

\section{BORIS CRASSINI \\ University of Queensland, \\ St. Lucia, Queensland 4067, Australia}

Spatially contingent color aftereffects (CAEs) like the McCollough effect (McCollough, 1965) decay more slowly than simple aftereffects (AEs) of tilt and motion. The differences in the temporal properties of CAEs and AEs have prompted investigators to propose explanations of CAEs in terms of more complex processes than simple neural adaptation thought to account for the occurrence of AEs (see Over, 1977; Stromeyer, 1978). In recent years, these "more complex" explanations of CAEs have begun to include "higher order, cognitive" mechanisms, and a number of novel experimental approaches (reviewed briefly in Broerse \& Crassini, 1980) have been used to provide support for these explanations. One such approach is based on the use of perceptually ambiguous figures (see Figure 1) as test stimuli following adaptation to typical McCollough-effect inspection patterns. For example, Jenkins and Ross (1977), Meyer and Phillips (1980), and Uhlarik, Pringle, and Brigell (1977) report correlations between fluctuations in the alternative subjective organizations of ambiguous test figures and fluctuations in the strength of CAEs (see also Meyer \& Sherman, 1981; Milewski, Iaccino, \& Smith, 1980). Despite their correlational nature, however, these data are interpreted as reflecting a causal relationship between the higher order, cognitive processes assumed to determine subjective perceptual organization and the mechanisms assumed to account for CAEs. In the present note, we question this explanation on the grounds that it unnecessarily presupposes a separation of poorly specified higher order cognitive processes, on the one hand, and lower order neural process, on the other. We suggest that a more parsimonious interpretation of the relationship between

Direct reprint requests to either Jack Broerse, Department of Psychology and Special Education, Mt. Gravatt College of Advanced Education, Messines Ridge Road, Mt. Gravatt 4122, Australia, or Boris Crassini, Department of Psychology, University of Queensland, St. Lucia, Queensland 4067, Australia.
CAE magnitude and fluctuations in perceptual organization is to consider both effects to involve similar, perhaps common, neural mechanisms. This approach is based on the assumption that fluctuations in perceptual organization of ambiguous figures can be considered as belonging to a wider class of perceptual phenomena involving alternations in subjective experience (e.g., monocular rivalry, Stromeyer, 1978, and Wade, 1975; binocular rivalry, Wade, 1975; disappearances and reappearances in geometrical patterns, Wade, 1977; and afterimages, Wade, 1978).

Before examining this claim in detail, a brief outline of the use of ambiguous figures as test stimuli for CAEs is warranted. Jenkins and Ross (1977), Meyer and Phillips (1980), and Uhlarik et al. (1977) induced CAEs in the usual manner (i.e., alternate inspection of, say, a vertical grating in red light, and a horizontal grating in green light). They then tested, with achromatic vertical and horizontal gratings arranged as a square with triangularly shaped components (Jenkins \& Ross, 1977; our Figure 1A), the traditional vase/faces figure (Meyer \& Phillips, 1980; our Figure 1B), or a diamond shaped component with a background (Uhlarik et al., 1977; and Figure 1C). Subjects reported CAEs when Figure 1A was perceived as a "bow-tie" of vertical contours, but not
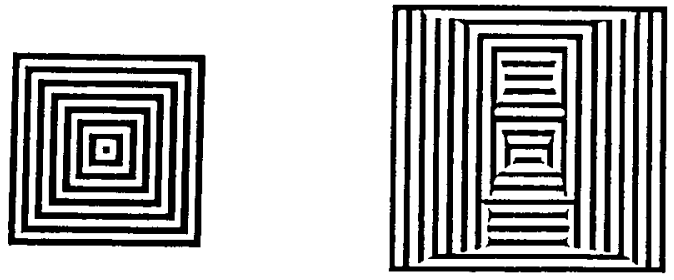

(A)

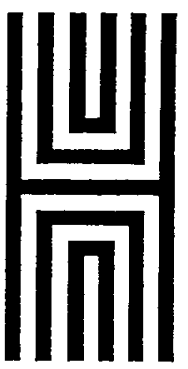

(C)

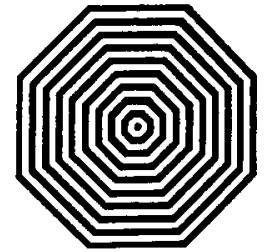

(D)
Figure 1. Examples of ambiguous figures used as test patterns following CAE induction. See text for detalls. 
when it was perceived as a set of concentric squares; subjects reported CAEs when Figure 1B was perceived as either a vase of horizontal contours against a background of vertical contours or two faces made up of vertical contours against a background of horizontal contours, but not when Figure 1B was perceived as concentric rectangles; and subjects reported CAEs when Figure $1 \mathrm{C}$ was perceived as a diamond of horizontal contours against a background of vertical contours, but not when it was perceived as a set of upright and inverted Us ("rectangles" in Uhlarik et al.'s terminology).

In all three reports, the investigators argue that, since CAEs appear and disappear as a function of alternation in the ambiguous figures, CAEs must be capable of being modified by the same factors that determine subjective perceptual organization. In all cases, the authors leave little doubt as to what the nature of such factors might be: "Our results show it is not only the dimensional values of the elements of the stimulus pattern which control the McCollough effect, but also the subjective imposed organization of the elements" (Jenkins \& Ross, 1977, p. 400); "the change in organization ... is not easily explainable by a passive or 'bottom-up' model of this perceptual situation (Meyer \& Phillips, 1980, pp. 604605); and "it must be assumed that cognitive factors can influence both the nature and extent that the underlying mechanisms ... are involved in the processing of pattern information" (Uhlarik et al., 1977, p. 510). Implicit in the "passive, bottom-up" vs. "active, top-down" approach adopted by Jenkins and Ross (1977), Meyer and Phillips (1980), and Uhlarik et al. (1977), and indeed in indirect theories of perception in general (see Turvey, 1977), is the notion that the organization of "degenerate" (to use Oatley's, 1978, term) sense data into subjective experience is mediated by "active, top-down" processes that are immune to factors that may influence lower level processes (e.g., neural adaptation to chromatic gratings). Although the authors cited do not use the word explicitly, there is a general feeling that "active, top-down" processes possess the property of intentionality and therefore are not stimulus dependent, while "passive, bottom-up" processes are involuntary and tied to properties of the stimuli in question.

Uhlarik et al. (1977) make this implication more explicit when they begin their discussion with the assertion: "The findings that subjective colors were generally not reported in the 'rectangles' organization of the test pattern, and that the mean duration of a 'rectangles' percept remained unchanged subsequent to adaptation to chromatic gratings, suggest that the 'rectangles' organization was unaffected by the experimental adaptation conditions"' (p. 509). However, on page 507, Uhlarik et al. state, "The finding of primary interest was that only the experimental (chromatic gratings) adaptation condition produced systematic changes in the tendencies to organize the test pattern." This statement is certainly supported by their data. Inspection of their Table 1 (shown in our Table 1) indicates that chromatic adaptation to gratings (unlike adaptation to achromatic gratings and contourless chromatic stimuli) resulted in a 13.6-sec increase in the total time the "diamond" organization was reported dominant for each of 27 observers, and also produced a decrease of 3.3 perceptual alternations averaged over these observers. The apparent contradiction between the quotes from pages 509 and 507 is resolved (?) when one considers that the statement on page 507 is based on the mean duration that the "rectangles" organization was dominant. This mean duration index is obtained by dividing the total duration by the number of alternations (i.e., sec/alternation) and seems to us to be an inappropriate (and artificial) index of perceptual stability. ${ }^{1}$ The raw data of total duration and number of alternations seem to us a more appropriate index of perceptual stability prior to and following chromatic adaptation. On the basis of these indices, the obvious conclusion from Uhlarik et al.'s data is that perceptual organization is altered by chromatic adaptation.

It is difficult to determine whether Uhlarik et al. (1977) are sensitive to this issue. Further on in their discussion, for example, these authors comment that "it is not surprising that the 'diamond' organization predominated following the experimental adaptation condition insofar as color differences between the

Table 1

Mean Pre- to Postadaptation Difference Scores (DS) and 95\% Confidence Limits (CL)

\begin{tabular}{|c|c|c|c|c|c|c|c|c|c|c|}
\hline & \multirow[b]{3}{*}{$\mathrm{n}$} & \multicolumn{3}{|c|}{ Total Amount of Time } & \multicolumn{4}{|c|}{ Duration } & \multirow{2}{*}{\multicolumn{2}{|c|}{$\begin{array}{c}\text { Number of } \\
\text { Switches }\end{array}$}} \\
\hline & & \multicolumn{2}{|c|}{ "Diamond" } & \multirow{2}{*}{$\begin{array}{l}\text { "Rectangles" } \\
\text { (DS) }\end{array}$} & \multicolumn{2}{|c|}{ "Diamond" } & \multicolumn{2}{|c|}{ "Rectangles" } & & \\
\hline & & DS & $\mathrm{CL}$ & & DS & $\mathrm{CL}$ & DS & $\mathrm{CL}$ & DS & $\mathrm{CL}$ \\
\hline Chromatic Grating & 27 & 13.6 & \pm 7.5 & -13.6 & 17.4 & \pm 11.2 & -1.8 & \pm 3.3 & -3.3 & \pm 2.0 \\
\hline Homogeneous Colors & 12 & 5.2 & \pm 8.9 & -5.2 & 3.2 & \pm 27.8 & -.8 & \pm 1.3 & -2.2 & \pm 2.6 \\
\hline Achromatic Gratings & 15 & .2 & \pm 8.9 & -.2 & -.4 & \pm 6.4 & .6 & \pm .8 & -1.7 & \pm 2.5 \\
\hline
\end{tabular}

Note-From "Color Aftereffects Contingent on Perceptual Organization" by J. Uhlarik, R. Pringle, \& M. Brigell, Perception \& Psychophysics, 1977, 22, 506-510. Copyright 1977 by the Psychonomic Society, Inc. Reprinted by permission. 
horizontal and vertical regions of this pattern would increase the salience of the central diamond on a surround of a different hue" (p. 509). However, in a postexperimental control condition, Uhlarik et al. do not pursue this issue. They add "real" desaturated colors to the ambiguous test figure in an attempt to mimic the CAE situation, but, instead of asking whether such real colors influence perceptual organization, they focus on whether the appearance of the real colors fluctuates with changes in perceived organization. The bias in direction of causation is clear: perceived organization may alter the appearance of colors (and is thus worthy of investigation), but the equally likely possibility that the colors may alter perceptual organization does not warrant investigation.

Nevertheless, it is apparent from Uhlarik et al.'s (1977) own data that adaptation to chromatic gratings results in both CAEs and changes in perceptual organization. While Jenkins and Ross (1977), Meyer and Phillips (1980), and Meyer and Sherman (1981) do not report their data in sufficient detail to make a comparable assessment, Milewski et al. (1980) have confirmed this finding. In the second of two experiments, Milewski et al. used an ambiguous test pattern (see Figure 1D) similar to that used by Jenkins and Ross (1977) but one that could be perceived as a set of concentric octagons or a set of eight triangular sections. Milewski et al. (1980) measured both changes in perceptual organization and color appearance of the test figure before and after adaptation to chromatic gratings, and concluded, "First, the aftereffect [CAE] strength itself can influence one perceptual organization over another. Second, the act of reorganizing a reversible figure can produce a decrement in aftereffect strength" (p. 336) ${ }^{2}$ The data reported by Uhlarik et al. (1977) and Milewski et al. (1980) cannot be accounted for by a model based on "passive, lower order processes" that can be influenced both by particular stimulus events (e.g., adaptation to chromatic gratings) and by "active, higher order" processes (which are themselves assumed to be immune to modification by such stimulus events). However, these data are consistent with a model in which CAE production and fluctuations in perceptual organization are considered to involve similar or common neural mechanisms equally susceptible to modification by, for example, adaptation to chromatic gratings.

This is by no means a new or radical proposition. Attneave (1976) reviewed a number of perceptual alternation phenomena ranging from the classic figural reversals of the Gestaltists to the visual art of Maurits C. Escher. After surveying a number of likely explanations, he attributes perceptual alternations to "activity in different neural structures, ... when one such structure becomes 'fatigued' or satiated or adapted, it gives way to another that is fresher or more excitable"' (p. 275). Attneave's (1976) explanation is similar to Hering's (1874/1964) account of binocular rivalry (perceptual alternations produced by dichoptic presentation of, say, orthogonal contours) and to more recent neural explanations of monocular rivalry (perceptual alternations produced with monocular or binocular viewing of orthogonal contours) (Campbell \& Howell, 1972; see Wade, 1977, 1975). Monocular rivalry, in particular, has been attributed to inhibition between "separate, and not significantly overlapping populations of neurons activated by gratings" (Campbell, Gilinsky, Howell, Riggs, \& Atkinson, 1973, p. 125).

Observations that spatial aftereffects that are dependent on color demonstrate similar form-color interactions to those found with monocular rivalry (Stromeyer, 1978) are consistent with the proposition that neural mechanisms with similar properties underlie both phenomena. In these terms, the finding that the durations of composite organizations during monocular rivalry (i.e., when both orientations are equally visible) decrease when complementary colors are paired with the orthogonal gratings (Wade, 1975) is comparable to the observations reported with ambiguous test patterns composed of horizontal and vertical components (as in Figure 1): When complementary colors (in this case, illusory colors produced by chromatic adaptation) are "paired" with these orthogonal components, the durations of subjectively organized composites (rectangles, squares, and octagons, see Figure 1) decrease significantly (see Table 1). While monocular rivalry involves patterns where orthogonal contours are superimposed, it seems plausible to suggest that the nonoverlapping orthogonal contours in the reversible patterns of Figure 1 involve inhibitory processes similar to those found with the superimposed orthogonal contours in the monocular rivalry situation.

In all the above explanations, perceptual alternations between different subjective organizations are considered in terms of inhibitory interactions between neural mechanisms simultaneously activated by stimulus components present in ambiguous figures. A corollary of this view is that subjective perceptual organization (and hence alternation) should be capable of being influenced by unambiguous stimulus events processed by the same neural mechanisms. Support for this hypothesis (in addition to the data of Milewski et al., 1980, and Uhlarik et al., 1977) is provided by Virsu's (1975) demonstration that prior inspection of an unambiguous Schroeder staircase (i.e., where only one organization is visible) influences perceptual organization during postinspection of an ambiguous Schroeder staircase (see, also, Emmerson, 1979).

When ambiguous figures composed of orthogonal achromatic gratings are used as test patterns, adaptation to orthogonal chromatic gratings simulta- 
neously produces CAEs as well as changes in the perceptual organization of the ambiguous figure. On the basis of these observations (illustrated in Uhlarik et al.'s data) and our discussion of perceptual effects demonstrating similar interactions between color and form, we conclude that explanations based on neural interactions have sufficient plausibility to reject assertions that a purely stimulus-bound model cannot provide a complete account of CAEs and related phenomena (e.g., perceptual alternation; see also Crassini, Broerse, \& O'Shea, 1979).

\section{REFERENCES}

Attne ave, F. Multistability in perception. In R. Held $\&$ W. Richards (Eds.), Recent progress in perception: Readings from Scientific American. San Francisco: Freeman, 1976.

Broerse, J., \& Crassini, B. The influence of imagery ability on color aftereffects produced by physically present and imagined induction stimuli. Perception \& Psychophysics, 1980, 28, 560568.

Campeell, F. W., Gilinski, A. S., Howell, E. R., Riggs, L. A., \& Atkinson, J. The dependence of monocular rivalry on orientation. Perception, 1973, 2, 123-125.

Campbell, F. W., \& Howell, E. R. Monocular alternation: A method for the investigation of pattern vision. Journal of Physiology, 1972, 225, 19-21.

Crassini, B., Broerse, J., \& O'Shea, R. P. Is the "contour specificity" of McCollough effects an example of anomalous transfer? Comments on Sharpe and Tees (1978). Perception \& Psychophysics, 1979, 25, 238-240.

Emmerson, P. L. Necker cube: Duration of preexposure of an unambiguous form. Bulletin of the Psychonomic Society, 1979, $14,397-400$.

Finke, R. A., \& Schmidt, M. J. Orientation-specific color aftereffects following imagination. Journal of Experimental Psychology: Human Perception and Performance, 1977, 3, 599-606.

Finke, R. A., \& Schmidt, M. J. The quantitative measure of pattern representation in images using orientation-specific color aftereffects. Perception \& Psychophysics, 1978, 23, 515-520.

Hening, E. Outline of a theory of the light sense (L. M. Hurvich \& D. Jameson, trans.). Cambridge, Mass: Harvard University Press, 1964. (Originally published, 1874.)

Jenkins, B., \& Ross, J. McCollough effect depends upon perceived organization. Perception, 1977, 6, 399-400.

McCollovgh, C. Color adaptation of edge-detectors in the human visual system. Science, 1965, 149, 1115-1116.

Meyer, G. E., \& Phillips, D. Faces, vases, subjective contours and the McCollough effect. Perception, 1980, 9, 603-606.

Meyer, G. E., \& Sherman, R. K. Reversible figures and the motion aftereffect. Vision Research, 1981, 21, 361-363.

Milewski, A. E., I Accino, J., \& SMITH, D. Checkerboardspecific color aftereffects: A failure to find effects of perceptual organization. Perception \& Psychophysics, 1980, 28, 329-336.

OntLey, K. Perceptions and representations. London: Methuen, 1978.
Over, R. Contingent aftereffects in the perception of color and contour. In R. H. Day \& G. V. Stanley (Eds.), Studies in perception. Perth: University of Western Australia Press, 1977.

Strome Yer, C. F. Form-color aftereffects in human vision. In R. Held, H. W. Leibowitz, \& H,-L. Teuber (Eds.), Handbook of sensory physiology (Vol. 8) Perception. Heidelberg: SpringerVerlag, 1978.

TurveY, M. T. Contrasting orientations to the theory of visual information processing. Psychological Review, 1977, 84, 67-88.

Uhlarix, J., Pringle, R., \& Brigell, M. Color aftereffects contingent on perceptual organization. Perception \& Psychophysics, 1977, 22, 506-510.

VIRsu, V. Determination of perspective reversals. Nature, 1975, 257, 786-787.

Wade, N. J. Monocular rivalry between contours. Perception, $1975,4,85-95$.

WADE, N. J. Distortions and disappearances of geometrical patterns. Perception, 1977, 6, 407-433.

WADE, N. J. Why do patterned afterimages fluctuate in visibility? Psychological Bulletin, 1978, 85, 338-352.

\section{NOTES}

1. Pre- to postadaptation differences in the mean total amount of time that any one organization is dominant accurately reflects overall changes in perceptual organization because one index is complementary to the other: Increases in one organization (e.g., "diamond") are accompanied by decreases in the other (e.g., "rectangles"). The mean duration between successive alternations for rectangles (the index used by Uhlarik et al.), however, is independent of the mean duration between successive alternations for diamonds. Such mean durations can stay constant despite large changes in the frequencies of occurrence for any one organization. Unless there is some specific hypothesis that predicts a change in the mean duration between alternations for any specific organization (e.g., "rectangles"), this index is inappropriate. Despite this point, had Uhlarik et al. pooled the durations between successive alternations for "diamond" and "rectangle" (see Table 1), overall changes in postadaptation perceptual organization reflected by this "pooled" index would be as large as the changes indicated by using the mean total amount of time. In both cases, the claim that the data reflect pre- and postadaptation stability is unreasonable.

2. While Milewski et al. (1980) acknowledge that Uhlarik et al.'s conclusions about the direction of influence between internal cognitive states and lower order neural activity are problematical, they do not reject "cognitive control" (their terminology) per se. They appear to advocate that the problem (i.e., of postadaptation changes in perceptual organization as well as color) lies in the choice of cognitive factors and suggest that these same factors (i.e., factors involving subjective organizations) are unable to account for the imagery-induced effects reported by Finke and Schmidt (1977, 1978; but see Broerse \& Crassini, 1980). They suggest, instead, that viewing and attentional strategies warrant further investigation.

(Manuscript received July 13, 1981; accepted for publication July 17,1981 .) 